\title{
ASSIGNMENT MODEL WITH MULTI-OBJECTIVE LINEAR PROGRAMMING FOR ALLOCATING CHOICE RANKING USING RECURRENT NEURAL NETWORK
}

\author{
Zahra Sadat Mirzazadeh ${ }^{1, *}$, Javad Bani Hassan ${ }^{1}$ And Amin Mansoori ${ }^{2}$
}

\begin{abstract}
Classic linear assignment method is a multi-criteria decision-making approach in which criteria are weighted and each rank is assigned to a choice. In this study, to abandon the requirement of calculating the weight of criteria and use decision attributes prioritizing and also to be able to assign a rank to more than one choice, a multi-objective linear programming (MOLP) method is suggested. The objective function of MOLP is defined for each attribute and MOLP is solved based on absolute priority and comprehensive criteria methods. For solving the linear programming problems we apply a recurrent neural network (RNN). Indeed, the Lyapunov stability of the proposed model is proved. Results of comparing the proposed method with TOPSIS, VICOR, and MORA methods which are the most common multi-criteria decision schemes show that the proposed approach is more compatible with these methods.
\end{abstract}

Mathematics Subject Classification. 90C29, 37N40, 37C75.

Received January 3, 2021. Accepted September 26, 2021.

\section{INTRODUCTION}

There are some choices in multi-criteria decision-making problems that should be assessed and ranked based on certain criteria set. The multi-criteria decision-making problem is usually shown with the following matrix called the decision matrix:

$$
D=\begin{array}{cccc}
C_{1} & C_{2} & \ldots & C_{n} \\
A_{1} \\
A_{2} \\
\vdots \\
A_{m}
\end{array}\left[\begin{array}{cccc}
x_{11} & x_{12} & \ldots & x_{1 n} \\
x_{21} & x_{22} & \ldots & x_{2 n} \\
\vdots & \vdots & \ddots & \vdots \\
x_{m 1} & x_{m 2} & \ldots & x_{m n}
\end{array}\right]
$$

Keywords. Linear assignment method, multi-objective linear programming, multi-attribute decision-making, absolute prioritizing method, recurrent neural networks, stable in the sense of Lyapunov.

1 Faculty of Sport Sciences, Ferdowsi University of Mashhad, Mashhad, Iran.

2 Department of Applied Mathematics, Ferdowsi University of Mashhad, Mashhad, Iran.

*Corresponding author: z.mirzazadeh@um.ac.ir

(C) The authors. Published by EDP Sciences, ROADEF, SMAI 2021 
where $A=\left\{A_{j} \mid 1 \leq j \leq m\right\}$ is the set of choices, $C=\left\{C_{j} \mid 1 \leq j \leq n\right\}$ is the set of criteria, and $x_{i j}$ shows the performance (score) of $i$ th choice based on $j$ th criteria. In order to express the relative importance of criteria, the weight $w_{j} \geq 0$ is assigned to the $j$ th criteria with the condition of $\sum_{j=1}^{n} w_{j}=1$. The purpose of analyzing multi-criteria decision-making problems is to rank and select the best choice from the set of choices based on the performance of each choice regarding all criteria; see [27]. Various methods have been developed and used for analyzing multi-criteria decision-making problems. However, all these methods include the following three steps:

1. Determining the set of criteria and choices;

2. Determining the numeric weight for criteria and performance (score) of each choice based on each criterion; 3. Processing numeric values of performances and weights to determine the rank of each choice [31].

There are so many methods for step 3, and classifying these methods is a popular research topic for many researchers. The reference [14] can be consulted to review and compare different multi-criteria decision-making methods. In the linear assignment method, choices of multi-criteria decision-making problems are ranked based on the performance of each choice regarding each criterion and the final choice ranking is obtained through a linear compensation process to combine and engage criteria. Only ordinal data are used as input in the linear compensation process and there is no need to convert qualitative data to quantitative ones or normalize data. The linear assignment method was first introduced as a programming model for selecting brands by consumers. The linear assignment model is a binary programming model and is used widely in solving assignment problems. Generally, assignment problem is a certain kind of transportation problem and it was formulated as a binary integer linear programming; see [18]. The main application of the standard assignment problem is solving the problem of assigning $n$ jobs to $n$ peoples in an optimum manner so that the minimum cost or maximum profit is obtained. Some such applications of the linear assignment model can be found in $[6,28]$, which are in the fields of urban service management, production schedule, traffic management, education planning, and navigation. Various algorithms, including linear programming, were used and developed to solve linear assignment problem; see $[3,29]$. The linear assignment model is originally formed to solve the problem of assigning $n$ jobs (machines) to $n$ persons (works) in an optimum manner, but, multi-criteria decision-making problem is used as one of the solutions and many of its applications are reported. There are $m$ ranks and $m$ choices in this application, so, the problem is finding the $i$ th choice for each of $m$ possible ranks with maximum effect on the rank. In addition, each rank should be assigned to only one choice and each choice should be gotten only one rank. Some applications of linear assignment model in solving multi-criteria decision-making problems can be found in $[1,32]$ covering topics such as the fuzzy assignment model for ranking export promotion plans, assessing competitive advantage of firms, selecting optimum maintenance policy, educational management, risk factor ranking, urban service management, classification, and fuzzy linear assignment model in transportation. In many real-life problems, decision-makers have more than one purpose. For example, those purposes include maximizing profit, minimizing costs, minimizing wastes, maximizing market share, and so on. Multi-objective linear programming (MOLP) deals with these kinds of problems [22]. MOLP is used to help decision-makers in complex problems when expressing the optimal condition is hard for them. As most real-life commercial decision-making problems aim more than one objective, an MOLP model is used in many fields and turned into a useful tool for decision-making; see [25]. Corresponding methods for solving MOLP have increased significantly. One such multi-criteria linear programming problem is optimizing a vector of linear functions while there are linear constraints. As there are several objective functions, the final answer is selected from a set of efficient points called efficient set. Some solutions of MOLP include target weighting, absolute priority, comprehensive criteria, and goal programming; see $[11,16]$. Today, MOLP is used in many fields including natural gas supply chain improvement, energy optimization, vehicle routing, and system classification; some of them can be found in references $[5,13]$.

Recurrent neural networks (RNNs) for solving constrained optimization problems can be considered as a tool to transfer the optimization problems into a specific dynamic model of first-order differential equations. The main idea of RNN approaches for a mathematical programming problem is to establish an energy function 
(nonnegative) and a dynamic system which is a representation of an RNN. An important requirement is that the energy function decreases monotonically as the dynamic system approaches an equilibrium point. The main advantage of RNN approach to optimization is that the nature of the dynamic solution procedure is inherently parallel and distributed. An RNN with a good computational performance should satisfy threefold. First, the global convergence of the proposed RNN with an arbitrarily given initial state should be guaranteed. Second, the network design preferably contains no variable parameter. Third, the equilibrium points of the network should correspond to the exact or approximate solution of the problem. From a mathematical point of view, these characteristics are relevant to the optimization techniques employed for deriving optimization RNN models. The pioneering works on an RNN model for optimization were done by Hopfield and Tank [15]. Neurodynamic optimization has received great success in recent years; see [8-10,19-21].

Motivated by the above discussion, the current study begins by reviewing the existing literature on linear assignment model for assigning choice ranking. Following the analysis of the shortcomings and definitions of the current patterns and methods, an RNN model will be developed for solving the linear assignment model. Moreover, the stability property of the model is proved.

The rest of the paper is organized as follows. Next section states the review of the literature on some methods for the problem. In Section 3, the material and methods of the paper are given. A new method based on MOLP for assigning choice ranking, an RNN model, and the stability of the RNN model are given in this section. Section 4 states the results and discussion. Finally, conclusions are provided in Section 5.

\section{LiteratuRE REVIEW}

In this section, we review the literature on some methods for the problem. In fact, we state the common pattern and also the shortcomings of the common pattern for some methods for solving the problem.

\subsection{Linear assignment model}

In the linear assignment, choices of multi-criteria decision-making problems are ranked based on their score regarding each criterion, and the final rank of each choice is obtained by using a linear compensation process, which is a combination and interaction of criteria. As only the performance difference of each pair of choices is considered regarding decision criteria, there is no need to convert qualitative data to quantitative one and to normalize data. The linear assignment model algorithm reads as follows (Algorithm 1).

\subsection{Absolute priority method}

Sometimes decision-maker does not want to determine the weight of the goal and assumes the goal weighting method to be a mental method. Indeed, they intend to prioritize goals. Therefore, the absolute priority method is deployed. In this method, the problem is solved only by a goal having first priority (more important) and the optimum answer is determined. Next, the objective function of first priority is put equal to the optimum result, and it is added to the problem as a constraint, and then we solve the problem by the objective function and the second priority. This procedure is repeated for subsequent priorities. The absolute priority method is used in most studies such that one objective is preferred to multiple objectives. The main issue in this method is that all objectives should be weighted; see [23].

\subsection{Comprehensive criteria method}

Unlike previous methods, there is no need to prioritize, give weight, or convert objectives to constraints. The comprehensive criteria method minimizes the total of the first exponent, the second exponent, and so on and the relative deviation of objectives from their optimum value depending on the case. In this method, an objective function that its minimization is always noteworthy is defined as follows:

$$
\min Z=\sum_{i=1}^{k}\left(f_{i}^{*}-\frac{f_{i}}{f_{i}^{*}}\right)^{p} .
$$






\subsection{Deficiencies of classic linear assignment method}

In the linear assignment method, first, weights should be calculated precisely because their exact value is needed to write the model as the exact extent of attributes' importance is not known in real conditions and it is required to calculate them - while attributes priority is easily determined by decision-makers - a linear programming method is proposed in this study to obtain a choice ranking without calculating the exact weight of attributes and by considering the priority of the attributes so that the opinion of decision-makers is also incorporated into the decision-making process. In addition, each choice is assigned only one rank and each rank is assigned to only one choice in a linear assignment method while in other methods of multi-attribute decisionmaking a rank can be assigned to more than one choice and two choices or more may have the same rank. Thus, a method is needed to overcome this weakness. Also, if it is necessary to add some additional attributes to a decision-making problem solved through the linear assignment method, the calculation should be performed from the beginning of the linear assignment method and even the weights of attributes should be recalculated, and this weakness is partly overcome in this study.

Comparing to the existence methods we summarized the advantages of the proposed method as the following two points:

1. The proposed method combines the classic linear assignment method, MOLP, the comprehensive criteria method or absolute priority method, and RNN. In fact, variables, objective function, and constraints are determine from the linear assignment method. Then an MOLP is constructed. 
2. We apply an RNN to solve the linear programming problems obtained from the previous sections. For the proposed RNN the stability and the convergence analysis theorems for the optimal solution are proved.

\section{MATERIALS AND METHODS}

Here, we are going to state the proposed method and the RNN model.

\subsection{Proposed method}

The method proposed in this study first determines variables the same as the classic linear assignment method. Then, an objective function maximizing the total of variables is defined per attribute to optimize choices regarding each decision attribute so that choices are optimized in terms of that attribute. Next, objective functions are defined for each attribute. Then, constraints of assigning only one rank to each choice and the binary condition of variables are added. Another constraint of the linear assignment model that points to assigning only one choice to each rank is not added to the model. Therefore, the classic linear assignment method is combined with MOLP. The resulted MOLP can be solved through the comprehensive criteria method or absolute priority method such that the final result for ranking choices is obtained. If the absolute priority method is used to solve the resulted MOLP, decision-makers should prioritize attributes that are obviously the same as objective function prioritizing. Therefore, the extent of attributes' (objectives) importance is effective without calculating the weight of attributes, and this model will be a flexible method in which the opinion of decision-makers influences the final ranking of choices. Consider a decision-making problem with choices $A_{i}$, $i=1,2, \ldots, m$, and attributes $C_{j}, j=1,2, \ldots, n$. Also $x_{i j}$ is the performance score of $i$ th choice regarding $j$ th attribute and the component of $i$ th row and $j$ th column in the decision matrix.

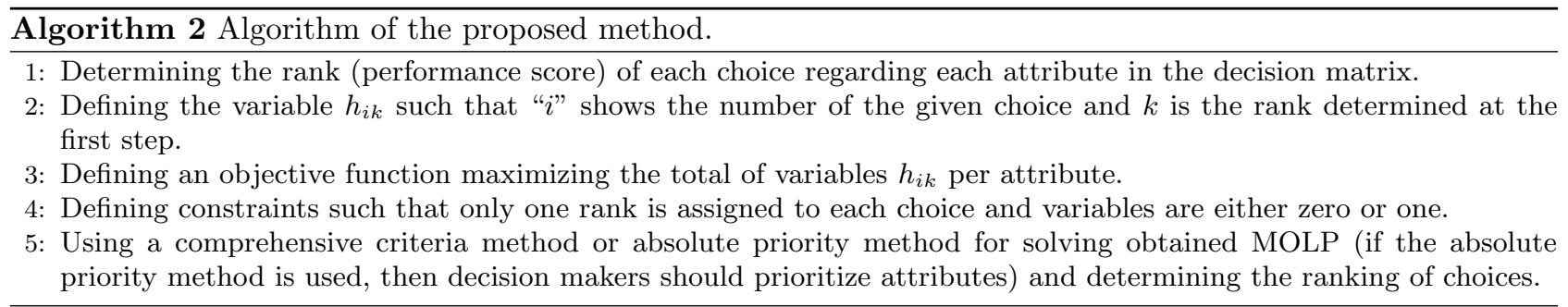

\subsection{RNN model}

In this section, an RNN model for solving the assignment problem with binary variables (2.2) is proposed. In fact, (2.2) is a linear programming problem with binary variables. Hence, here we consider the general form of the linear programming problem with binary variables. Consider the general form of (2.2) as follows:

$$
\begin{aligned}
\min & c^{T} x \\
\text { s.t. } & A x=b, \\
& x \in\{0,1\} .
\end{aligned}
$$

Because of the total unimodality property of the constraint coefficient matrix defined in (2.2) [2], the integrality constraint in the $(2.2)$ can be equivalently replaced with the nonnegativity constraint. In fact, in the original problem if a unique optimum exists, then we can transfer the zero-one constraint with the nonnegativity constraint; see [2]. By the above discussion, the following equivalent linear programming problem can be 
formulated:

$$
\begin{array}{cl}
\min & c^{T} x \\
\text { s.t. } & A x=b, \\
& x \geq 0 .
\end{array}
$$

The Lagrangian of (3.2) is constructed by

$$
L(x, u, v)=c^{T} x-\frac{1}{2} u^{2} x+v(A x-b),
$$

where $u$ and $v$ are the Lagrange multipliers. Thereby, the KKT (Karush-Kuhn-Tucker) optimality conditions are given as:

$$
\text { KKT conditions: }\left\{\begin{array}{l}
u^{*}, x^{*} \geq 0, u^{*} x^{*}=0, \\
c^{T} x^{*}-u^{*}+A^{T} v^{*}=0, \\
A x^{*}=b .
\end{array}\right.
$$

Since (3.2) is a linear optimization so the duality gap is zero and thus, the KKT optimality conditions are both the necessary and the sufficient conditions for optimality. Now, our goal is to describe an RNN model satisfying the KKT optimality conditions. In accordance with the convexity of $(3.2)$, the gradient of $L(\cdot, \cdot, \cdot)$ must be zero at the optimal point. Thus, it provides:

$$
\left\{\begin{array} { l } 
{ \nabla _ { x } L ( x , u , v ) = 0 , } \\
{ \nabla _ { u } L ( x , u , v ) = 0 , } \\
{ \nabla _ { v } L ( x , u , v ) = 0 , }
\end{array} \Longrightarrow \left\{\begin{array}{l}
-\left(c-\frac{1}{2} u^{2}+A^{T} v\right)=0, \\
-\operatorname{diag}(u) x=0, \\
A x-b=0
\end{array}\right.\right.
$$

Here, our aim is to construct a continuous-time dynamical system that will settle down to the KKT point of linear programming problem (3.2). Therefore, our aim now is to design an RNN model that will settle down to the saddle point of $L(x, u, v)$. Note that, the problem (3.2) is a convex optimization problem so the gradient of $L(x, u, v)$ with respect to the variables $x, u$, and $v$ must be vanished at $\left(x^{*}, u^{*}, v^{*}\right)$. Therefore, based on (3.5), we describe the RNN model for solving the original problem (3.2) as the following nonlinear dynamical system:

$$
\begin{aligned}
\frac{\mathrm{d} x}{\mathrm{~d} t} & =-\left(c-\frac{1}{2} u^{2}+A^{T} v\right), \\
\frac{\mathrm{d} u}{\mathrm{~d} t} & =-\operatorname{diag}(u) x, \\
\frac{\mathrm{d} v}{\mathrm{~d} t} & =A x-b .
\end{aligned}
$$

For simplifying the proposed RNN model, we define

$$
\Theta(y)=\Theta(x, u, v)=\left[\begin{array}{c}
-\left(c-\frac{1}{2} u^{2}+A^{T} v\right) \\
-\operatorname{diag}(u) x \\
A x-b
\end{array}\right] .
$$

Then the proposed model can be summarized as follows:

$$
\frac{\mathrm{d} y}{\mathrm{~d} t}=\zeta \Theta(y)
$$

where $\zeta>0$ is the convergence rate. Thus, employing previous discussion we have the following theorem.

Theorem 3.1. $\left(x^{*}, u^{*}, v^{*}\right)^{T}$ is the optimal solution of (3.4), if and only if, $y^{*}$ is the equilibrium point of the RNN model (3.9).

Proof. The proof follows from the previous discussion. 


\subsection{Theoretical analysis}

Here, we study the stability analysis of the proposed model (3.9). We start this subsection with some necessary requirements from dynamical system [17].

Definition 3.2 (Equilibrium point). In the following dynamical system:

$$
\dot{x}=f(x(t)), \quad x\left(t_{0}\right)=x_{0} \in \mathbb{R}^{n},
$$

where $f$ is a function from $\mathbb{R}^{n}$ to $\mathbb{R}^{n}$ and $x^{*}$ is called an equilibrium point of (3.10), if $f\left(x^{*}\right)=0$.

Definition 3.3 (Stability in the sense of Lyapunov). Suppose that $x(t)$ is a solution for (3.10). The equilibrium point $x^{*}$ is said to be stable in the sense of Lyapunov, if for any $x_{0}=x\left(t_{0}\right)$ and any $\varepsilon>0$, there exists $\delta>0$, such that

$$
\left\|x(t)-x^{*}\right\|<\varepsilon, \quad \text { for all } t \geq t_{0}, \quad\left\|x\left(t_{0}\right)-x^{*}\right\|<\delta .
$$

Definition 3.4 (Lyapunov function). Let $\Omega \subseteq \mathbb{R}^{n}$ be an open neighborhood of $x^{*}$. A continuously differentiable function $E: \mathbb{R}^{n} \longrightarrow \mathbb{R}$ is said to be a Lyapunov function at the state $x^{*}$ over the set $\Omega$ for (3.10), if

$$
\begin{cases}E\left(x^{*}\right)=0, E(x)>0, & \text { for all } x \in \Omega \backslash\left\{x^{*}\right\} \\ \frac{d E(x(t))}{\mathrm{d} t}=\nabla E(x(t))^{T} f(x(t)) \leq 0, & \text { for all } x \in \Omega .\end{cases}
$$

Lemma 3.5. Assume that $f: \mathbb{R}^{n} \rightarrow \mathbb{R}^{n}$ is a continuous mapping. Then, for arbitrary $t_{0} \geq 0$ and $x_{0} \in \mathbb{R}^{n}$, there is a local solution $x(t)$ for the dynamical system (3.10) with $t \in\left[t_{0}, \tau\right)$ for some $\tau \geq t_{0}$. Furthermore, if $f$ is locally Lipschitzian continuous at $x_{0}$, then the solution is unique, and if $f$ is Lipschitzian continuous in $\mathbb{R}^{n}$, then $\tau$ can be extended into $\infty$.

Lemma 3.6. If a mapping $F$ is continuously differentiable on an open convex set $D$ including $\Omega$, then $F$ is monotone (strictly monotone) on $\Omega$, if and only if, $\nabla F(x)$ is positive semi-definite (positive definite) for all $x \in \Omega$.

Now, we prove a lemma stating the uniqueness solution for RNN model (3.9).

Lemma 3.7. There exists a unique solution $y(t)$ for the model (3.9).

Proof. As $c-\frac{1}{2} u^{2}+A^{T} v$ and all parts of the proposed model are locally Lipschitz continuous, then, by employing Lemma 3.5, the proof over $\left[t_{0}, \tau\right)$ for some $\tau>t_{0}$ as $\tau \rightarrow \infty$ is completed.

The main result of this section (i.e., the stability and the convergence theorem for model (3.9)) is stated in next theorem.

Theorem 3.8. The proposed model (3.9) is stable in the sense of Lyapunov.

Proof. Theorem 3.1 and Lemma 3.7 give the existence and the uniqueness of the solution of $(3.9)$ over $\left[t_{0}, \infty\right)$. Assume the following Lyapunov function:

$$
E(y)=e^{\left(\|\Theta(y)\|^{2}+\frac{1}{2}\left\|y-y^{*}\right\|^{2}\right)}-1 .
$$

The Jacobian matrix $\nabla \Theta(y)$ with some calculation can be obtained as follows:

$$
\nabla \Theta(y)=\left[\begin{array}{ccc}
0 & \operatorname{diag}(u) & -A^{T} \\
-\operatorname{diag}(u) & -\operatorname{diag}(x) & 0 \\
A & 0 & 0
\end{array}\right]
$$


TABLE 1. Quantitative data for the problem of selecting flexible manufacturing system (decision matrix).

\begin{tabular}{llllllll}
\hline \hline \multicolumn{7}{c}{ Attributes } \\
\hline Choices & $C_{1}^{+}$ & $C_{2}^{+}$ & $C_{3}^{+}$ & $C_{4}^{+}$ & $C_{5}^{+}$ & $C_{6}^{-}$ & $C_{7}^{-}$ \\
\hline$A_{1}$ & 30 & 23 & 5 & 0.745 & 0.745 & 1500 & 5000 \\
$A_{2}$ & 18 & 13 & 15 & 0.745 & 0.745 & 1300 & 6000 \\
$A_{3}$ & 15 & 12 & 10 & 0.5 & 0.5 & 950 & 7000 \\
$A_{4}$ & 25 & 20 & 13 & 0.745 & 0.745 & 1200 & 4000 \\
$A_{5}$ & 14 & 18 & 14 & 0.255 & 0.745 & 950 & 3500 \\
$A_{6}$ & 17 & 15 & 9 & 0.745 & 0.5 & 1250 & 5250 \\
$A_{7}$ & 23 & 18 & 20 & 0.5 & 0.745 & 1100 & 3000 \\
$A_{8}$ & 16 & 8 & 14 & 0.255 & 0.5 & 1500 & 3000 \\
\hline
\end{tabular}

and also,

$$
\nabla \Theta(y)^{T}+\nabla \Theta(y)=\left[\begin{array}{ccc}
0 & 0 & 0 \\
0 & -2 \operatorname{diag}(x) & 0 \\
0 & 0 & 0
\end{array}\right]
$$

On the other hand, $\frac{\mathrm{d} \Theta}{\mathrm{d} t}=\frac{\partial \Theta}{\partial y}^{T} \frac{\mathrm{d} y}{\mathrm{~d} t}=\zeta \nabla \Theta(y)^{T} \Theta(y)$. Also, we have $E\left(y^{*}\right)=0$, and for $y \neq y^{*}$, so $E(y)>0$, and

$$
\begin{aligned}
& \frac{\mathrm{d}}{\mathrm{d} t} E(y)=\frac{\partial}{\partial t} E(y) \frac{\mathrm{d} y}{\mathrm{~d} t} \\
& =\left[\left(\frac{\mathrm{d} \Theta}{\mathrm{d} t}\right)^{T} \Theta(y)+\Theta(y)^{T} \frac{\mathrm{d} \Theta}{\mathrm{d} t}+\left(y-y^{*}\right)^{T} \frac{\mathrm{d} y}{\mathrm{~d} t}\right] \exp \left(\|\Theta(y)\|^{2}+\frac{1}{2}\left\|y-y^{*}\right\|^{2}\right) \\
& =\zeta\left[\Theta(y)^{T} \nabla \Theta(y)^{T} \Theta(y)+\Theta(y)^{T} \nabla \Theta(y) \Theta(y)+\left(y-y^{*}\right)^{T} \Theta(y)\right] e^{\left(\|\Theta(y)\|^{2}+\frac{1}{2}\left\|y-y^{*}\right\|^{2}\right)} \\
& =\zeta\left[\Theta(y)^{T}\left(\nabla \Theta(y)^{T}+\nabla \Theta(y)\right) \Theta(y)+\left(y-y^{*}\right)^{T} \Theta(y)\right] e^{\left(\|\Theta(y)\|^{2}+\frac{1}{2}\left\|y-y^{*}\right\|^{2}\right)} \\
& =\zeta\left[-2(\operatorname{diag}(u) x)^{2} x+\left(y-y^{*}\right)^{T} \Theta(y)\right] e^{\left(\|\Theta(y)\|^{2}+\frac{1}{2}\left\|y-y^{*}\right\|^{2}\right)} \\
& \leq \zeta\left[-2(\operatorname{diag}(u) x)^{2} x\right] e^{\left(\|\Theta(y)\|^{2}+\frac{1}{2}\left\|y-y^{*}\right\|^{2}\right)} \leq 0 .
\end{aligned}
$$

As $x \geq 0$, therefore, $-2(\operatorname{diag}(u) x)^{2} x \leq 0$. Thus, $\frac{\mathrm{d}}{\mathrm{d} t} E(y)<0$ except for $y \neq y^{*}$. Therefore, applying Theorem 2.2 from [17], the model (3.9) is stable in the sense of Lyapunov.

\section{Results And Discussion}

In this section, a problem is presented taken from Chakraborty [4]. In this example, there are eight choices assessed regarding 7 criteria. Octoploid choices are flexible manufacturing systems. Criteria include: reducing labor costs, $C_{1}$; reducing work in progress, $C_{2}$; reducing implementation costs, $C_{3}$; increasing market responsiveness, $C_{4}$; quality optimization, $C_{5}$; investment and maintenance costs, $C_{6}$; and required space, $C_{7}$. The codes for RNN model (3.9) are presented by using interpreted computer language MATLAB and the computations are performed on a system with Intel core 7 Duo processor $2 \mathrm{GHz}$ and 4 GB RAM. Table 1 shows the performance (score) of each choice per criteria.

The weight of each criterion is calculated through the entropy method. Then, the single-objective linear programming model resulted from the classic linear assignment method is formed after determining the rank of each choice regarding each 8 attributes and forming the reference matrix $\Lambda_{m \times m}$ with nonnegative elements 
TABLE 2. Variables of linear assignment method for numerical example.

\begin{tabular}{llllllll}
\hline \hline \multicolumn{7}{c}{ Attributes } \\
\hline Choices & $C_{1}^{+}$ & $C_{2}^{+}$ & $C_{3}^{+}$ & $C_{4}^{+}$ & $C_{5}^{+}$ & $C_{6}^{-}$ & $C_{7}^{-}$ \\
\hline$A_{1}$ & $h_{11}$ & $h_{11}$ & $h_{17}$ & $h_{11}$ & $h_{11}$ & $h_{16}$ & $h_{14}$ \\
$A_{2}$ & $h_{24}$ & $h_{25}$ & $h_{22}$ & $h_{21}$ & $h_{21}$ & $h_{25}$ & $h_{26}$ \\
$A_{3}$ & $h_{37}$ & $h_{36}$ & $h_{35}$ & $h_{32}$ & $h_{32}$ & $h_{31}$ & $h_{37}$ \\
$A_{4}$ & $h_{42}$ & $h_{42}$ & $h_{44}$ & $h_{41}$ & $h_{41}$ & $h_{43}$ & $h_{43}$ \\
$A_{5}$ & $h_{58}$ & $h_{53}$ & $h_{53}$ & $h_{53}$ & $h_{51}$ & $h_{51}$ & $h_{52}$ \\
$A_{6}$ & $h_{65}$ & $h_{64}$ & $h_{66}$ & $h_{61}$ & $h_{62}$ & $h_{64}$ & $h_{65}$ \\
$A_{7}$ & $h_{73}$ & $h_{73}$ & $h_{71}$ & $h_{72}$ & $h_{71}$ & $h_{72}$ & $h_{71}$ \\
$A_{8}$ & $h_{86}$ & $h_{87}$ & $h_{83}$ & $h_{83}$ & $h_{82}$ & $h_{86}$ & $h_{81}$ \\
\hline & & & & & & &
\end{tabular}

showing the effect of $A_{i}, i=1,2, \ldots, 8$, for the $k$ th rank, $k=1,2, \ldots, 8$. The optimum result of the linear assignment method for this example is obtained from the RNN model (3.9) as:

$$
h_{11}=h_{28}=h_{37}=h_{44}=h_{53}=h_{65}=h_{72}=h_{86}=1,
$$

and other variables are zero. According to step 5 of linear assignment model, the final rating of choices is as follows:

$$
A_{2}<A_{3}<A_{8}<A_{6}<A_{4}<A_{5}<A_{7}<A_{1} .
$$

Now, a numerical example is solved with the method proposed in this study:

Step 1: Determining the rank (performance score) of each choice regarding each criterion in the decision matrix. Step 2: Defining the variable $h_{i k}$ such that $i$ is the number of given choice and $k$ is the rank determined at step 1 and shown in Table 2 .

Step 3: Defining an objective function maximizing total of variables $h_{i k}$ per criteria as follows:

$$
\begin{aligned}
\max & h_{11}+h_{24}+h_{37}+h_{42}+h_{58}+h_{65}+h_{73}+h_{86} \\
& h_{11}+h_{25}+h_{36}+h_{42}+h_{53}+h_{64}+h_{73}+h_{87} \\
& h_{17}+h_{22}+h_{35}+h_{44}+h_{53}+h_{66}+h_{71}+h_{83} \\
& h_{11}+h_{21}+h_{32}+h_{41}+h_{53}+h_{61}+h_{72}+h_{83} \\
& h_{11}+h_{21}+h_{32}+h_{41}+h_{51}+h_{62}+h_{71}+h_{82} \\
& h_{16}+h_{25}+h_{31}+h_{43}+h_{51}+h_{64}+h_{72}+h_{86} \\
& h_{14}+h_{26}+h_{37}+h_{43}+h_{52}+h_{65}+h_{71}+h_{81}
\end{aligned}
$$

Step 4: Defining constraints such that each choice is assigned only one rank and variables are either zero or one:

$$
\begin{array}{ll}
\text { s.t. } & \sum_{k=1}^{m} h_{i k}=1, \quad i=1,2, \ldots, m, \\
& h_{i k}= \begin{cases}1 & \text { if rank } k \text { assigns to the choice } A_{i} \\
0 & \text { if rank } k \text { does not assign to the choice } A_{i} .\end{cases}
\end{array}
$$

Step 5: Using the comprehensive criteria method or absolute priority method for solving the obtained MOLP model (if an absolute priority method is used, then decision makers should prioritize attributes) and determining choices' rank. 
Optimum answer of the example by using the proposed method with comprehensive criteria method (as the purpose of this study is introducing a linear programming method, comprehensive criteria method with $P=1$ is used) and RNN model (3.9) is as follows:

$$
h_{11}=h_{25}=h_{37}=h_{42}=h_{53}=h_{65}=h_{71}=h_{86}=1 .
$$

And other variables are zero. Then, the final ranking of the choices is as follows:

$$
A_{3}<A_{8}<A_{6}<A_{2}<A_{5}<A_{4}<A_{7}<A_{1} .
$$

Optimum answer of the example using the proposed method with absolute priority method if decision makers have the following attributes' prioritizing:

$$
C_{7}<C_{6}<C_{5}<C_{4}<C_{3}<C_{2}<C_{1}
$$

applying RNN model (3.9), is as follows:

$$
h_{11}=h_{24}=h_{37}=h_{42}=h_{58}=h_{65}=h_{73}=h_{86}=1 .
$$

And other variables are zero. Then, the final rank of choices is as follows:

$$
A_{5}<A_{3}<A_{8}<A_{6}<A_{2}<A_{7}<A_{4}<A_{1} .
$$

Comparing rankings resulted from the classic linear assignment and the method proposed in this study shows that the choice ranking is different. There is no constraint of assigning one rank to two or more choices in our proposed method, and as it can be seen in the ranking, resulted from the proposed method of this study in which comprehensive criteria method is used to solve its MOLP, rank one is assigned to two choices, which are first and seventh choices, and rank five is assigned to two choices, which are second and sixth choices. In the proposed method of this study, the exact weight of criteria is not used, but attributes' priority is used. Now, if it is needed to add another attribute to the existing one and the problem is to be solved through the classic linear assignment method, then the weight of criteria should be recalculated and the linear assignment method should be run from the beginning. However, the proposed method only needs to form the variables related to that attribute and its objective function should be added to the MOLP model and MOLP is resolved. It can be seen that calculations are significantly decreased in the proposed method.

It is expected that the ranking through the proposed method is more credible than the classic linear assignment method. To investigate it, the results can be compared with several multi-criteria methods. It seems that TOPSIS, VIKOR, and MOORA are appropriate in this regard as TOPSIS is currently one of the most common methods for multi-criteria decision-making that ranks choices based on minimizing the distance from the ideal point and maximizing it from anti-ideal point $[7,30]$. The VIKOR method is also a common decision-making method in which choices are ranked based on the existing attributes such that they would be nearer to the ideal [24] and the MOORA method is known as a credible decision-making method in which ranking is performed after normalizing decision matrix using the software and then calculating the attribute (weight value) of each choice $[4,26]$. Rankings resulted from TOPSIS, VIKOR, and MOORA, linear assignment, and the proposed method of this study are presented in Table 3. In order to compare the proposed method with the classic linear assignment method, Spearman's rank correlation coefficient [12] was calculated between three methods of TOPSIS, VIKOR, MOORA, and classic linear assignment method, and also between three methods of TOPSIS, VIKOR, MOORA and the method proposed in this study and all of them are shown in Table 4. As you can see in the table, the correlation of classic linear assignment method with MOORA method is 0.73 and the correlation of the proposed method that its linear programming is solved by absolute priority method, with MOORA method is 0.67 ; but, the proposed method is more correlated with decision-making methods of TOPSIS, VIKOR, and MOORA than the classic linear assignment method. Then, the proposed method of this study is more compatible with three methods of TOPSIS, VIKOR, and MOORA than the classic linear assignment method. 
TABLE 3. Ranking resulted from different methods for numerical example.

\begin{tabular}{lcccccccc}
\hline \hline & \multicolumn{1}{c}{ Choices } \\
\hline Method & $A_{1}$ & $A_{2}$ & $A_{3}$ & $A_{4}$ & $A_{5}$ & $A_{6}$ & $A_{7}$ & $A_{8}$ \\
\hline TOPSIS method & 4 & 3 & 8 & 2 & 6 & 5 & 1 & 7 \\
VIKOR method & 3 & 4 & 7 & 2 & 5 & 6 & 1 & 8 \\
MOORA method & 3 & 5 & 8 & 2 & 4 & 6 & 1 & 7 \\
Linear assignment method & 1 & 8 & 7 & 4 & 3 & 5 & 2 & 6 \\
Proposed method (Absolute priority) & 1 & 4 & 7 & 2 & 8 & 5 & 3 & 6 \\
Proposed method (Comprehensive criteria) & 1 & 5 & 7 & 2 & 3 & 5 & 1 & 6 \\
\hline
\end{tabular}

TABLE 4. Spearman's rank correlation coefficient between three methods of TOPSIS, VIKOR, and MOORA with linear assignment method and the proposed method.

\begin{tabular}{lccc}
\hline \hline & \multicolumn{3}{c}{ Method 2 } \\
\hline Method 1 & TOPSIS method & VIKOR method & MOORA method \\
\hline $\begin{array}{l}\text { Linear assignment method } \\
\begin{array}{l}\text { Proposed method with } \\
\text { Absolute priority }\end{array}\end{array}$ & 0.40 & 0.59 & 0.73 \\
$\begin{array}{l}\text { Proposed method with } \\
\text { Comprehensive criteria }\end{array}$ & 0.76 & 0.73 & 0.67 \\
\hline
\end{tabular}

\section{Conclusions}

In the current study, a multi-criteria decision-making method was introduced in which an MOLP is presented in a way that one linear objective function is defined for each decision-making attribute. There is no need to calculate the weight of criteria in this method and criteria's priority is used. In the proposed method, model variables such as variables of the classic linear assignment method were defined but there is no constraint of assigning only one choice to one rank. In addition, by adding new attributes to the problem, only some objective function was added to the MOLP of the problem, and calculations were significantly reduced compared with the linear assignment method. For solving the linear programming problems, an RNN model was developed. Also, the stability of the model was proved. We solved the proposed RNN model by an ODE. Here, we reformulated the KKT conditions of the linear programming problem into an efficient RNN model to solve the problem. The Spearman rank correlation coefficient was calculated between the linear assignment method and the proposed method with three methods of TOPSIS, VIKOR, and MOORA that shows that the proposed method is more credible than the classic linear assignment method and it can be a better replacement for the classic linear assignment method. Like other multi-criteria decision-making methods in assessing and ranking choices, the method proposed in this study can be used in many different contexts.

In this study, a linear programming method was used that maximizes the total of variables. A nonlinear programming model can be used in future studies, or bounded variables or integer variables can be used by changing the method of defining variables. In this study, absolute priority and comprehensive criteria method were used to solve the MOLP model so other methods can be used in future studies. Also, as data are not necessarily crisp in the real world, the proposed method can be developed for fuzzy data.

\section{REFERENCES}

[1] A. Baykasoglu, K. Subulan and F.S. Karaslan, A new fuzzy linear assignment method for multi-attribute decision making with an application to spare parts inventory classification. Appl. Soft Comput. 42 (2016) 1-17. 
[2] M.S. Bazaraa, C. Shetty and H.D. Sherali, Linear programming and network flows. John Wiley \& Sons, New York (1990).

[3] R. Burkard, M. Dell'Amico and S. Martello, Assignment problems. Society for Industrial Mathematics (2009).

[4] S. Chakraborty, Applications of the MOORA method for decision-making in manufacturing environment. Int. J. Adv. Manuf. 54 (2011) 1155-66.

[5] V.M. Charitopoulos and V. Dua, A unified framework for model-based multi-objective linear process and energy optimization under uncertainty. Appl. Energy 186 (2017) 539-548.

[6] M.M. Dessouky and B.A. Kijowski, Production scheduling on single-stage multiproduct batch chemical process with fixed batch sized. IIE Trans. 29 (1997) 399-408.

[7] L. Dymova, P. Sevastjanov and A. Tikhonenko, An interval type-2 fuzzy extension of the TOPSIS method using alpha cuts. Knowl. Based Syst. 83 (2015) 116-127.

[8] S. Effati, A. Mansoori and M. Eshaghnezhad, An efficient projection neural network for solving bilinear programming problems. Neurocomputing 168 (2015) 1188-1197.

[9] M. Eshaghnezhad, S. Effati and A. Mansoori, A neurodynamic model to solve nonlinear Pseudo-Monotone projection equation and its applications. IEEE Trans. Cybern. 47 (2017) 3050-3062.

[10] M. Eshaghnezhad, F. Rahbarnia, S. Effati and A. Mansoori, An artificial neural network model to solve the fuzzy shortest path problem. Neural Process. Lett. 50 (2019) 1527-1548.

[11] A.A. Foroughi and Y. Jafari, A modified method for constructing efficient solutions structure of MOLP. Appl. Math. Model. 33 (2009) 2403-10.

[12] T.D. Gauthier, Detecting trends using spearman's rank correlation coefficient. Environ. Forensics 2 (2001) 359-362.

[13] S. Ghosh and S.K. Roy, Fuzzy-rough multi-objective product blending fixed-charge transportation problem with truck load constraints through transfer station. RAIRO: OR 55 (2021) S2923-S2952.

[14] M. Hasan, I.E. Büyüktahtakın and E. Elamin, A multi-criteria ranking algorithm (MCRA) for determining breast cancer therapy. Omega 82 (2019) 83-101.

[15] J.J. Hopfield and D.W. Tank, Neural computation of decisions in optimization problems. Biol. Cybern. 52 (1985) $141-152$.

[16] A.H. Hosseinian and V. Baradaran, A multi-objective multi-agent optimization algorithm for the multi-skill resourceconstrained project scheduling problem with transfer times. RAIRO: OR 55 (2021) 2093-2128.

[17] H.K. Khalil, Nonlinear systems. Prentice-Hall, Michigan (1996).

[18] M. Khorbatly, H. Dkhil, H. Alabboud and A. Yassine, A hybrid algorithm Tabu Search - GRASP for wounded evacuation in disaster response. RAIRO: OR 54 (2020) 19-36.

[19] A. Mansoori and M. Erfanian, A dynamic model to solve the absolute value equations. J. Comput. Appl. Math. 333 (2018) $28-35$.

[20] A. Mansoori and M. Mohammadi, A Projection Neural Network for Identifying Copy Number Variants. IEEE J. Biomed. Health Inform. 23 (2019) 2182-2188.

[21] A. Mansoori, M. Eshaghnezhad and S. Effati, Recurrent Neural Network Model: A New Strategy to Solve Fuzzy Matrix Games. IEEE Trans. Neural Netw. Learn. Syst. 30 (2019) 2538-2547.

[22] R.T. Marler and J.S. Arora, Survey of multi-objective optimization methods for engineering. Struct. Multidiscipl. Optim. 26 (2004) 369-95.

[23] G. Minella, R. Ruiz and M. Ciavota, A review and evaluation of multi objective algorithms for the owshop scheduling problem. INFORMS J. Comput. 20 (2007) 451-471.

[24] M. Omidvar and F. Nirumand, An extended VIKOR method based on entropy measure for the failure modes risk assessment - A case study of the geothermal power plant (GPP). Saf. Sci. 92 (2017) 160-172.

[25] H. Omrani, S. Mohammadi and A. Emrouznejad, A bi-level multi-objective data envelopment analysis model for estimating profit and operational efficiency of bank branches. RAIRO: OR 53 (2019) 1633-1648.

[26] J.D. Patel and K.D. Maniya, Application of AHP/MOORA method to select wire cut electrical discharge machining process parameter to cut EN31 alloys steel with brasswire. Mater. Today: Proc. 2 (2015) 2496-2503.

[27] J. Rezaei, Best worst multi criteria decision-making method. Omega 53 (2015) 49-57.

[28] S.A. Sadabadi, A. Hadi-Vencheh, A. Jamshidi and M. Jalali, A linear programming technique to solve fuzzy multiple criteria decision making problems with an application. RAIRO: OR $\mathbf{5 5}$ (2021) 83-97.

[29] A. Schrijver, Combinatorial Optimization, Polyhedra and efficiency, Algorithms and combinatorics. Springer Verlag, Berlin, 24 (2003).

[30] L. Sun, C.L. Miao and L. Yang, Ecological-economic efficiency evaluation of green technology innovation in strategic emerging industries based on entropy weighted TOPSIS method. Ecol. Indic. 73 (2017) 554-558. 
[31] E. Triantphyllou, Multi Criteria Decision Making Methods: Comparative Study. Dordrecht, Kluwer Academic Publisher (2000).

[32] S. Wang, O. Meng and C.Y. Lee, Liner container assignment model with transit-time-sensitive container shipment demand and its applications. Transport. Res. Part B: Meth. 90 (2016) 135-155.

\section{Subscribe to Open (S20) A fair and sustainable open access model}

This journal is currently published in open access under a Subscribe-to-Open model (S2O). S2O is a transformative model that aims to move subscription journals to open access. Open access is the free, immediate, online availability of research articles combined with the rights to use these articles fully in the digital environment. We are thankful to our subscribers and sponsors for making it possible to publish this journal in open access, free of charge for authors.

\section{Please help to maintain this journal in open access!}

Check that your library subscribes to the journal, or make a personal donation to the S2O programme, by contacting subscribers@edpsciences.org

More information, including a list of sponsors and a financial transparency report, available at: https://www. edpsciences.org/en/maths-s2o-programme 